\title{
EVALUATION OF PROTECTIVE EFFECTS OF SOLUBLE EGG ANTIGEN CONJUGATED WITH TREHALOSE DIMYCOLATE ADJUVANT IN EXPERIMENTAL SCHISTOSOMIASIS MANSONI
}

\section{By}

\author{
HEBA SABRY SHABAAN, OMAIMA KAMEL EL-SHAFEY, BAHAA EL-DEEN \\ WADE EL-ASWAD, SHAIMAA AHMED SHARAF EL-DEEN, \\ And REHAM MUSTAFA BRAKAT*.
}

Department of Parasitology, Faculty of Medicine, Menoufia University, Shebin ElKom, Egypt ( ${ }^{*}$ Correspondence: reham_brakat@yahoo.com Mobile: 00201004774422)

\section{Abstract}

Schistosomiasis is the second most common parasitic infection all over the world. It is responsible for thousands of deaths per year. Control of this infection depends mainly on praziquantel which unfortunately has many defects e.g. occurrence of reinfection and risk of resistance. So, a suitable vaccine is an urgent need. The vaccination that elicits a combined Th1 and Th2 is considered a proper vaccine candidate. Trehalose Dimycolate (TDM) is an immunogen that proved potent activation of Th1 lymphocytes. Effects of its combination with the Th2 stimulant, soluble egg antigen (SEA) in protection against Schistosoma mansoni infection in experimental animals were studied. Combined SAE+TDM achieved statistically significant protection against infection causing reduction of the worm load and ova count. A large percentage of the laid ova were even immature. Combined immunization was associated with reduced hepatic granuloma number, diameter and hepatic fibrosis percentage. Moreover, SEA+TDM increased stimulation of M2 type macrophages that limited the spread of hepatic fibrosis. It was concluded that SEA+TDM achieved the best protection from infection and the resulting pathology. This can be regarded to increased activation of humoral and cellular wings of immunity and activations of both anti-S. mansoni and tissue protecting macrophages and cytokines.

Key Words: Soluble Egg Antigen, Trehalose Dimycolate, Schistosoma mansoni.

\section{Introduction}

Schistosomias is the second cause of death due to parasitic infections of humans after malaria (Keitel et al, 2019). A recent Global Burden of Disease report documented that 190 million people are infected with schistosomes, with 70 million new infections and thousands of deaths occurring annually (Global Burden of Disease Study, 2017). World Health Organization reported that schistosomiasis was responsible for more than 21 thousand deaths in the Sub-Saharan Africa of 24,068 deaths globally (WHO, 2018). Moreover, it was one of the diseases included within the WHO's 2020 goals for neglected tropical diseases (NTD) control (WHO, 2013). Estimates showed that at least 229 million people required preventive treatment; at least $90 \%$ of them live in Africa (WHO, 2020).

Schistosomiasis control is usually achieved by periodic large-scale preventive chemotherapy of the infected populations using praziquantel (WHO, 2020). Despite that, this control measure has many limitations e.g. high rates of reinfection, the potential development of drug-resistant strains (Pinto-Almeida et al, 2016), requirement of a large infrastructure to cover all parts of an endemic area and associated costs (Stephenson et al, 2014). That is why the combination of $P Z Q$ with an anti-schistosomiasis vaccine was considered the best long-term control strategy (Martin et al, 2012). Protective vaccination against schistosomiasis required potent activation of both Th1 \& Th2 lymphocytes to cover all the disease phases (McManus and Loukas, 2008). In natural infection, Th1 lymphocytes prevail in the first two to five weeks of infection followed by Th2 started by stimulation of antigens of deposited ova (Pearce and MacDonald, 2002). That's why many egg antigens were appropriate candidates for vaccine trials, which mainly drive a potent $\mathrm{Th} 2$ response (Everts et al, 2009). To increase the potency of immune response or 
stimulate more immune cells, the selection of a suitable adjuvant is a critical step in the path to develop a successful anti-Schistosoma vaccine. Incorporation of an immunologically potent adjuvant can enhance and prolong qualified immune reactions to used vaccines (Vogel, 2000, Etewa et al, 2014).

Trehalose-6,6'-dimycolate (TDM), known as cord factor, is an abundant glycolipid in the mycobacterial cell wall (Bloch, 1950), which stimulated Th1 \& Th17 responses (Decout et al, 2017) with subsequent induction of a wide range of cytokines e.g. IL-12, IFN- $\gamma$, TNF- $\alpha$, IL-4, IL-6 \& IL-10 (Ryll et al, 2001).

The present work aimed to assess the value of combining TDM adjuvant with SEA in protection against Schistosoma mansoni infection of experimental animals and the possible mechanisms of this protection.

\section{Materials and Methods}

Ethics Statement: All animal experiments were performed at Theodor Bilharz Research Institute (TBRI), Giza, Egypt. Mice were kept under standard housing conditions in the animal house of TBRI and were maintained on a standard commercial pelleted diet in an air-conditioned room at $20-22^{\circ} \mathrm{C}$. All experimental procedures were performed in accordance with the international ethical guidelines after approval of the institutional ethical committee of TBRI.

Study Design: 50 Male pathogen-free BALB/c mice (6-8 weeks, 18-20gm) were equally divided among five groups. Group (GI) served negative control. Group (GII) served as positive control for $S$. mansoni. Group (GIII) was immunized by SEA and then infected with S. mansoni. Group (GIV) was immunized by TDM adjuvant and then infected with S. mansoni. Group (V) given combined SEA and TDM immunization and then infected with $S$. mansoni.

Trehalose Dimycolate adjuvant: The TDM Adjuvant (Sigma-Aldrich, USA, the Sigma Adjuvant System (Catalog Number S6322) was used for mice immunization of GIV \& GV. The immunization protocol was per- formed according to the manufacturer's instructions. Briefly, each mouse was subcutaneously (S.C.) injected with $200 \mu$ l of TDM $(100 \mu$ in each flank). Same dose was repeated once after 2 weeks as a booster dose.

Soluble egg Antigen preparation (SEA): Soluble egg antigen (SEA) was purchased from Schistosome Biological Supply Centre at TBRI. Concentration was adjusted with phosphate-buffered saline (PBS) to be $20 \mu \mathrm{g} /$ $\mathrm{ml}$ and was stored at $-70^{\circ} \mathrm{C}$ until used. The immunization protocol was performed (Nabih and Soliman, 1986). Mice of the GIII and GV were immunized with an initial S.C. injection with $30 \mu \mathrm{g}$ of the SEA and a booster dose of $20 \mu \mathrm{g}$ of the SEA with a time interval of 2 weeks.

Schistosoma mansoni infection: One week after the last booster dose, mice of the last 4 groups were S.C. injected with $80-100$ cercariae suspended in $0.2 \mathrm{ml}$ of distilled water (Peters and Warren, 1969).

Euthanizing mice and sample collection: One week after the first dose and one week after the booster dose of immunogens, blood samples were collected from the retroorbital sinus (Teixeira de Melo et al, 2013). Serum was separated by centrifugation of the collected blood samples at $3000 \mathrm{rpm}$ for 5 min and kept at $-20^{\circ} \mathrm{C}$ until use. At the $7^{\text {th }}$ week post-infection, mice were euthanized by decapitation and underwent removal of livers and intestines after counting worms. Each liver was divided into 2 equal parts; one was fixed in formalin $10 \%$ for further histopathological studies. Second was digested in $\mathrm{KOH} \mathrm{5 \%}$ to count the ova.

Evaluation of the vaccine protective effects:

A- Worm load, worm recovery was made by normal saline perfusion of hepatic and porto-mesenteric vessels through cannulation of the inferior vena cava of euthanized mice. Male, female and coupled worms were counted (Duvall and DeWitt, 1967). Retrieved adult worms were preserved in glutaraldehyde for further electron microscopic studies.

B- Tissue egg load, samples of liver and 
intestine (ileum) were collected from each mouse, weighed and digested in $5 \% \mathrm{KOH}$ at $37^{\circ} \mathrm{C}$ for 16 hours. Ova were counted at $\mathrm{x} 40$ magnifications (Herbert et al, 2010).

Oogram pattern: Intestinal segments were opened lengthwise and the excess mucus was removed. One-centimeter fragments were weighed and placed between a glass slide and a glass cover. Preparation was inverted and pressed on a rubber surface padded with filter paper. Percentages of immature, mature, and dead ova in the small intestines were computed from a total of 100 eggs/intestinal segment and classified according to categories (Pellegrino et al, 1962).

SEM of adult worms: Retrieved adults were fixed with $2.5 \%(\mathrm{v} / \mathrm{v})$ glutaraldehyde in PBS, ( $\mathrm{pH} \mathrm{7.4)} \mathrm{for} 24$ hours at room temperature. Later, they were rinsed three times with PBS before storage in PBS at $4^{\circ} \mathrm{C}$ until use. Before SEM, adult worms were washed twice with distilled water, dehydrated in ascending ethanol concentrations, and critically point dried. They were put on aluminium stubs, sputter coated with $20 \mathrm{~nm}$ gold nanoparticles, and examined in a high-resolution SEM (Jol 5200 lv, Japan) at an accelerating voltage of $5 \mathrm{kV}$ in Electron Microscope Unit, Faculty of Agriculture, Mansoura University (Manneck et al, 2011).

Hematoxylin and eosin staining of granuloma: Formalin preserved liver samples were dehydrated in ascending grades of alcohol i.e. $70,80,90, \& 100 \%$ for $30 \mathrm{~min}, 40$ $\min \& 1$ hr respectively then washed with xylene for $1 \mathrm{hr}$. Dehydrated tissues were embedded in molten paraffin wax. Tissues were cut into thin sections, mounted on clean glass microscopic slides and stained with hematoxylin and eosin stain ( $\mathrm{H} \& \mathrm{E}$ stain). Slides were blot-dried, mounted with Distyrene Plasticizer Xylene (Goyal et al, 2013). Granulomas were counted, and their diameter was digitally measured using a multihead microscope, Olympus SC100 and, analySIS getIT software. The diameter of granuloma with a single ovum was only considered ones.
Masson's trichrome staining of hepatic fibrosis: Paraffinized liver samples were stained using Masson's trichrome staining kit, (Sigma-Aldrich, Egypt). Staining procedures were done according to the manufacturer's instructions. Analysis of fibrosis was applied to photos for all fields of each slide. Percent of fibrosis was calculated using image $\mathrm{J}$ software program version $1.47 \mathrm{v}$ (Amin and Mahmoud-Ghoneim, 2011).

Immunohistochemical staining of arginase-1 \& inducible nitric oxide synthase (iNOS) markers of macrophages: Immune staining of paraffinized liver sections was done using anti-arginase- 1 and anti- iNOS antibodies (Abcam, USA) as given by Kim et al. (2014). Positivity was confirmed when the cell membrane and/or the cytoplasm showed brown staining. Stained slides were evaluated quantitatively using the H-score. Briefly, the intensity of staining was given a number from $(1+, 2+\& 3+)$ for mild, moderate and strong staining respectively. Percentage of stained cells in each tissue was multiplied by the intensity of staining. A score of 0-300 was given for the stained biomarker according to the following equation $\mathrm{H}$ score $=[1 \times$ $(\%$ cells $1+)+2 \times(\%$ cells $2+)+3 \times(\%$ cells 3+)] (Fraser et al, 2003).

ELISA for detection of specific IgG, IgG1, IgG2a, IFN- $\gamma$ \&, IL-10: Serum levels of specific IgG, IgG1 and IgG2a were measured in serum samples by homemade ELISA technique (El-Aswad et al, 2019). Serum levels of IFN- $\gamma$ and IL-10 were measured at the 2 times intervals before infection using a solid-phase sandwich ELISA which were purchased from Life Technologies Corporation for IFN- $\gamma$ and Abcam for IL-10. Procedures were performed according to the manufacturer's protocol. OD values were measured at an absorbance of $450 \mathrm{~nm}$ with an ELISA reader (Bio-Rad, UK).

Statistical analysis: Data entry, coding, and analysis were conducted using SPSS (20), IBM Corp. Released 2011: IBM SPSSStatistics for Windows; Version 20.0. Armonk, NY: IBM Corp. The data were of the 
quantitative type, and were expressed in Mean and Standard Deviation (SD). The degree of protection (\% reduction of worm) by the various immunogens was calculated as follows: $\mathrm{P}(\% \mathrm{R})=\mathrm{C}-\mathrm{V} / \mathrm{C} \times 100$, where $\mathrm{P}$ was the percentage protection, $\mathrm{C}$ is the mean number of parasites recovered from infected mice and $\mathrm{V}=$ mean of parasite recovered from vaccinated mice (Tendler et al, 1986). ANOVA and Kruskal Wallis tests were used to estimate the difference between means of groups of parametric and non-parametric data respectively. Post Hoc Value was used to assess the difference in two means of two individual groups. The interpretation of Post Hoc test was as follows:

p1: comparison between infected GII and SEA immunized (GIII). p2: comparison between infected (GII) and TDM immunized (GIV). p3: comparison between infected (II) group and SEA + TDM immunized group (V). p4: comparison between SEA immunized (GIII) and TDM immunized (GIV). p5: comparison between SEA immunized (GIII) and SEA+TDM immunized (GV). P6: comparison between TDM immunized (GIV) and SEA + TDM immunized (GV). Level of significance of the present data was $95 \%$, so, p-value $>0.05$ was not considered as a significant difference, while $\mathrm{p}$-value $<0.05$ was considered a significant difference.

\section{Results}

The highest worm load reduction percentage either in male, female or coupled worms was detected in mice vaccinated with SEA \&TDM, those vaccinated with sole SEA or TDM with a statistically significant difference between them (P5<0.05*, P6 < $0.001 * *)$ (Tab. 1).

The ultrastructural changes, adults retrieved from SEA+TDM immunized mice showed the highest incidence of multiple damages in the form of extensive tegmental damage, surface blebbing, oedema, rupture, vacuolation, necrosis and lost tubercular spines. Deformed suckers and gynaecophoric canals were also detected. Female worms showed furrowing, loss of spines, poring, fissuring, surface cracking and blebbing (Fig. 1).

The highest percent of reductions of tissue egg load-either that of the intestine or liver tissues, and the oogram were detected in mice groups vaccinated with SEA \&TDM then those vaccinated with SEA. The lowest reduction percentage was in TDM vaccinated mice with statistically significant differences between all vaccinated groups (Fig. 2).

The histopathological examination of liver tissues showed the highest percent of reductions of hepatic granuloma number, diameter and fibrosis in mice vaccinated with SEA \&TDM than sole SEA or TDM vaccinated mice with statistically significant differences between vaccinated groups (Figs. $3 \& 4$ ).

Both types of macrophages were assessed in the present work. Alternatively, activated macrophages (i.e. M2 type) expressing Arginase- 1 were the highest in mice group that received a combined immunization of SEA and TDM followed be sole SEA immunized mice with a statistically significant difference between both groups $(\mathrm{p}<0.05)$. The reverse occurred regarding iNOS expressing classically activated macrophages (i.e. M1 type), which recorded the lowest $\mathrm{H}$-score in SEA+TDM group followed by sole SEA group. The difference was also a statistically significant one $(\mathrm{p}<0.05)$ (Figs. $5 \& 6)$.

As to ELISA, the highest mean values of specific IgG, IgG1, IgG2a, in both times of their assessment, were recorded in mice immunized with combined SAE+TDM followed by sole SEA immunized mice with a statistically significant difference between both groups $(\mathrm{p}<0.05)$. Like immunoglobulins, the immune-regulating cytokine, IL-10 recorded the highest scores when SEA was used in combination with TDM in both times of its assessment. The reverse was recorded with the pro-inflammatory cytokine, INF-y that was the lowest with SEA+TDM combination. Differences were all statistically significant $(\mathrm{p}<0.05)$ (Fig. 7). 
Table 1: Comparison between means of adult worm load in groups.

\begin{tabular}{|c|c|c|c|c|c|c|}
\hline Worm load & Groups & Mean \pm SD & Reduction \% & Significance test & $\mathrm{P}$ value & Post hoc test \\
\hline Male worms & $\begin{array}{l}\text { GII (+ve control) } \\
\text { GIII (SEA) } \\
\text { GIV (TDM) } \\
\text { GV (SEA+TDM) }\end{array}$ & $\begin{array}{l}5.00 \pm 1.15 \\
1.5 \pm 0.49 \\
3.1 \pm 0.99 \\
0.50 \pm 0.52\end{array}$ & $\begin{array}{l}---- \\
70 \% \\
38 \% \\
90 \%\end{array}$ & $\mathrm{~K}=32.45$ & $<0.001$ & $\begin{array}{l}\text { p1: }<0.001 \\
\text { p2: }<0.05 \\
\text { p3: }<0.001 \\
\text { p4: }<0.05 \\
\text { p5: }<0.05 \\
\text { p6: }<0.001\end{array}$ \\
\hline $\begin{array}{l}\text { Female } \\
\text { worms }\end{array}$ & $\begin{array}{l}\text { GII (+ve control) } \\
\text { GIII (SEA) } \\
\text { GIV (TDM) } \\
\text { GV (SEA+TDM) }\end{array}$ & $\begin{array}{l}4.00 \pm 1.15 \\
1.2 \pm 0.91 \\
2.4 \pm 0.69 \\
0.2 \pm 0.35\end{array}$ & $\begin{array}{l}---- \\
70 \% \\
40 \% \\
95 \%\end{array}$ & $K=29.94$ & $<0.001$ & $\begin{array}{l}\text { p1: }<0.001 \\
\text { p2: }<0.05 \\
\text { p3: }<0.001 \\
\text { p4: }<0.05 \\
\text { p5: }<0.05 \\
\text { p6: }<0.001\end{array}$ \\
\hline $\begin{array}{l}\text { Coupled } \\
\text { worms }\end{array}$ & $\begin{array}{l}\text { GII (+ve control) } \\
\text { GIII (SEA) } \\
\text { GIV (TDM) } \\
\text { GV (SEA+TDM) }\end{array}$ & $\begin{array}{l}10.00 \pm 1.24 \\
3.6 \pm 0.69 \\
5.1 \pm 1.69 \\
2.1 \pm 0.73\end{array}$ & $\begin{array}{l}----- \\
64 \% \\
49 \% \\
79 \%\end{array}$ & $\mathrm{~F}=85.79$ & $<0.001$ & $\begin{array}{l}\text { p1: }<0.001 \\
\text { p2: }<0.001 \\
\text { p3: }<0.001 \\
\text { p4: }<0.05 \\
\text { p5: }<0.05 \\
\text { p6: }<0.001\end{array}$ \\
\hline $\begin{array}{l}\text { Total } \\
\text { worms }\end{array}$ & $\begin{array}{l}\text { GII (+ve control) } \\
\text { GIII (SEA) } \\
\text { GIV (TDM) } \\
\text { GV (SEA+TDM) }\end{array}$ & $\begin{array}{l}29.40 \pm 0.84 \\
10.00 \pm 1.70 \\
14.40 \pm 1.17 \\
8.20 \pm 0.63\end{array}$ & $\begin{array}{l}------- \\
65.9 \% \\
51.02 \% \\
72.11 \%\end{array}$ & $\mathrm{~F}=689.15$ & $<0.001$ & $\begin{array}{l}\text { p1: }<0.001 \\
\text { p2: }<0.001 \\
\text { p3: }<0.001 \\
\text { p4: }<0.001 \\
\text { p5: }<0.05 \\
\text { p6: }<0.001\end{array}$ \\
\hline
\end{tabular}

$\mathrm{K}=$ kruskal wallis test, $\mathrm{F}=$ ANOVA test, $\mathrm{p} 1$ : compared between GII \& GIII. p2: compared between GII \& GIV. p3: compared between GII \& GV. p4: compared between GIII \& GIV.p5: comparison between GIII \& GV. p6: compared between GIV \& GV.

\section{Discussion}

Worm load reduction is the gold standard for anti-Schistosoma vaccine development (McManus and Loukas, 2008) so, worm load was assessed in our study and it revealed that combination of TDM with SEA achieved the highest percent of worm load reduction either in male, female or coupled worms. These results can be explained by the ultrastructural changes detected in SEA + TDM group. Adults retrieved from SEA +TDM immunized mice showed the highest incidence of multiple damages. Tegumental destruction rendered the worm liable to the immune system attacks (Abdel-Zaher et al, 2016), which were increased by the tested immunogens as explained later. Moreover, deformed tubercles and suckers decrease adherence of the worms to the mesenteric blood vessels, so they can be washed back easily to be destroyed. This was similar to what was reported by El-Ahwany et al. (2012) and Etewa et al. (2018) who regarded these morphological changes to SEA which acts similar to the actual infections, because it is a crude antigen, where primary infec- tions can inhibit secondary ones. Reduction percentage re-ached $90 \%$ with the combination of TDM makes was a good candidate according to Nascimento et al. (2002) who defined good vaccine as the one able to achieve more than $40 \%$ of worm load reduction.

Reduction of the adults' load was reflected on both hepatic and intestinal ova numbers where the highest oreduction percentage was detected with combined SEA \& TDM. This can be regarded to deforme gynecophoric canal with expected abnormal matting and thus decreased female maturation and egg deposition (Steinauer, 2009). Also, Oogram patterns gave a hint that combining TDM with SEA exceeded affecting the number of ova to the quality of the laid ova. The percentage of immature ova and dead ones were higher than the sole SEA immunized group with a statistically significant difference between both groups. The reverse was detected regarding the mature ones.

The reduced ova count was reflected on number of hepatic granulomas where the lowest number of granulomas was detected 
in the mice that received SEA+TDM combination. The effect of TDM exceeded the number of granulomas to include its diameter and percentage of the occurring hepatic fibrosis. SAE+TDM group achieved the lowest scores with a statistically significant difference when compared with sole SEA immunized mice. These results can be explained by Etewa et al. (2017 \& 2019) who explained the reduction in number and size of liver granulomatous reactions by the SEA-induced specific stimulation of the immune reactions against Schistosoma eggs which reduces hepatic pathology and fibrosis. The additive effect of TDM can be explained by Sharma et al., (1985) who reported reduced pathological changes of Entamoeba histolytica in rabbits immunized by TDM+ crude Entamoeba antigen.

Because macrophages are one of the main cellular constituents of granulomas and critical regulators of liver fibrosis (Zhu et al, 2014; Abdel-Zaher et al, 2016), both types of macrophages were assessed in the present work. Alternatively, activated macrophages (i.e. M2 type) expressing Arginase-1 were the highest in mice group received a combined immunization of SEA \& TDM. The reverse occurred in iNOS expressing classically activated macrophages (i.e. M1 type) that recorded the lowest $\mathrm{H}$-score in SEA+ TDM group followed by sole SEA group.

Studies revealed that SEA increases activation of the alternatively activated macrophages i.e. M2 type. This type is responsible for the Schistosoma-induced fibrosis via the metabolism of 1-arginine to proline and polyamine by Arginase-1 so, it down regulated inflammatory responses and facilitated tissue remodeling (Barron and Wynn, 2011; Zhu et al, 2014). However, TDM is responsible for the activation of the classical macrophages (i.e. M1 type) through stimulation of Th1 lymphocytes with increased IFN- $\gamma$ mediated M1 polarization. M1 macrophages are activated in the early stage of Schistoso$m a$ infection and kill schistosomula by producing nitric oxide (Ahmed et al, 1997). Al- so, they play a role in preventing hepatic fibrosis (Hesse et al, 2001; Barron and Wynn, 2011; Decout et al, 2017). So, combined immunization achieved benefits of both macrophages by decreasing both granulomas and fibrosis. M2 was more predominant because of its suppressive ability to M1 macrophages (Sica and Mantovani, 2012).

The highest mean values of specific IgG, IgG1 \& IgG2a, in both times of their assessment, were recorded in mice immunized with combined SAE+TDM. Although TDM stimulates the Th1 induced IgG2a production (Ryll et al, 2001), it enhanced the SEA mediated shifting to IgG 1 (Gaber et al, 2010) thus combination resulted in synergistic action to the SEA rather than the additive effect. Like immunoglobulins, the immuneregulating cytokine, IL-10 recorded highest scores when SEA was used in combination with TDM in both times of its assessment. Reverse was recorded with pro-inflammatory cytokine, INF-y that was the lowest with SEA+TDM combination. IL-10 played an important role in schistosomiasis by preventing the development of Th1 \& Th2-mediated pathologies. IL-10 reduces hepatocyte damage induced by the eggs of Schistosoma, so it was essential for the maintenance of a non-lethal chronic infection and inhibits inappropriate immune responses (El-Ahwany et al, 2012; Stephenson et al, 2014).

\section{Conclusion}

A combination of TDM to SEA immunogen achieved the best protection from infection and the resulting pathology. This can be regarded to increased activation of humoral and cellular wings of immunity and activations of both anti-S. mansoni and tissue protecting macrophages and cytokines.

Declarations of interest: The authors declared that they neither have special interest nor receive fund.

\section{Acknowledgements}

The authors expressed their thanks to Dr. Shereen Fathy, Assistant Professor of Pathology for her kind efforts in liver sections examination and the results interpretation. 


\section{References}

Abdel-Zaher, M, Abed, GH, Abdel-Hakeem, SS, 2016: Ultrastructural changes of Schistosoma mansoni worms associated with administration of its polyvalent vaccine. J. Zool. St. 3, 6:920.

Ahmed, SF, Oswald, IP, Caspar, P, Hieny, S, Keefer, L, et al, 1997: Developmental differences determine larval susceptibility to nitric oxide-mediated killing in a murine model of vaccination against Schistosoma mansoni. Infect. Immun. 65:219-26.

Amin, A, Mahmoud-Ghoneim, D, 2011: Texture analysis of liver fibrosis microscopic images: A study on the effect of biomarkers. Acta. Biochim. Biophys. Sin. (Shanghai). 43, 3:193203.

Barron, L, Wynn, TA, 2011: Macrophage activation governs schistosomiasis-induced inflammation and fibrosis. Eur. J. Immunol. 41:250914.

Bloch, H, 1950: Studies on the virulence of tubercle bacilli: The relationship of the physiological state of the organisms to their pathogenicity. J. Exp. Med. 92, 6:507-26.

Decout, A, Silva-Gomes, S, Drocourt, D, Barbe, S, André, I, et al, 2017: Rational design of adjuvants targeting the C-type lectin Mincle. P.N.A.S. 114, 10:2675-80.

Duvall, RH, DeWitt, WB, 1967: An improved perfusion technique for recovering adult schistosomes from laboratory animals. Am. Trop. Med. Hyg. J. 16: 483e486 (PMID: 4952149).

El-Aswad, BEW, Harba, NM, Moharm, IM, Mahmoud, SF, 2019: Immunization with adult Schistosoma mansoni tegument, treated with sub-curative praziquantel, partially protects mice against the infection. J. Helminthol. 1-12. https://doi.org/10.1017/S0022149X18001207

El-Ahwany, E, Bauiomy, IR, Nagy, F, Zalat, R, Mahmoud, O, et al, 2012: Regulatory cell respon-ses to immunization with a soluble egg antigen in Schistosoma mansoni-infected mice. Korean J. Parasitol. 50, 1:29-35.

Etewa, SE, Abd El-Aal, NF, Abdel-Rahman, SA, El-Shafey, MA, 2014: Parasitological evaluation of potential candidate vaccines in Schistosoma mansoni-infected mice. J. Parasitol. Vector Biol. 6, 2:23-30.

Etewa, SE, Al-Hoot, AA, Sharaf, HM, Moawad, HSF, Samira, M, et al, 2019: Modeling approaches to predict and evaluate schistosomiasis immunization utilizing SEA loaded on chitosan nanoparticles via liver tissue differentiation and angiogenesis. P.U.J. 12, 3:1687-92.

Etewa, SE, Hegab, MHA, Metwally, AS, AbdAllah, SH, Shalaby, SM, et al, 2017: Murine hepatocytes DNA changes as an assessment of the immunogenicity of potential anti-schistosomal vaccines experimentally. J. Parasit. Dis. 41, 1:219-29.

Everts, B, Perona-Wright, G, Smits, HH, Hokke, CH, van der Ham, A, et al, 2009: Omega1 , a glycoprotein secreted by Schistosoma mansoni eggs, drives Th2 responses. J. Exp. Med. 206. 8:1673-80.

Fraser, JA, Reeves, JR, Stanton, PD, Black, D M, Going, JJ, et al, 2003: A role for BRCA1 in sporadic breast cancer. Br. Cancer J. 88, 8:1263e 1270.http://dx.doi.org/10.1038/sj.bjc. 6600863 .

Gaber, HM, Maghraby, AS, Ahmed, MB, Ruppel, A, Bahgat, MM, 2010: Immune responses in mice after immunization with antigens from different stages of the parasite Schistosoma mansoni. Z. Naturforsch. C. J. Biosci. 65:289-302. Global Burden of Disease Study (GBD Collaborators) 2017: Global, regional, and national incidence, prevalence, and years lived with disability for 328 diseases and injuries for 195 countries, 1990-2016: A systematic analysis for the global burden of disease (GBD) Study 2016. Lancet 390:1211-59.

Goyal, N, Rishi, P, Shukla, G, 2013: Lactobacillus rhamnosus GG antagonizes Giardia intestinalis induced oxidative stress and intestinal disaccharidases: An experimental study. World Microbiol. Biotechnol. J. 29, 6:1049-57.

Herbert, DR, Orekov, T, Roloson, A, Ilies, M, Perkins, C, et al, 2010: Arginase 1 suppresses IL-12/IL-23p40-driven intestinal inflammation during acute schistosomiasis. Immunol. J. 184, 11:6438-46.

Hesse, M, Modolell, M, La Flamme, AC, Schito, M, Fuentes, JM, et al, 2001: Differential regulation of nitric oxide synthase- 2 and arginase1 by type 1/type 2 cytokines in vivo: Granulomatous path-ology is shaped by the pattern of 1arginine metabolism. J. Immunol. 167:6533-44. Keitel, WA, Potter, GE, Diemert, D, Bethony, J, El Sahly, HM, et al, 2019: A phase 1 study of the safety, reactogenicity, and immunogenicity of a Schistosoma mansoni vaccine with or without glucopyranosyl lipid A aqueous formulation (GLA-AF) in healthy adults from a non- 
endemic area. Vaccine 37:6500-9.

Kim, HS, Jung, YY, Do, SI, 2014: Hepatic inducible nitric oxide synthase expression increases upon exposure to hypergravity. Braz. J. Med. Biol. Res. 47, 11:940-6.

Manneck, T, Braissant, O, Ellis, W, Keiser, J, 2011: Schistosoma mansoni: Antischistosomal activity of the four optical isomers and the two racemates of mefloquine on schistosomula and adult worms in vitro and in vivo. Exp. Parasitol. 127, 1:260-9.

Martin, VP, Pinheiro, CS, Figueiredo, BCP, Assis, NRG, Morais, SB, et al, 2012: Vaccination with enzymatically cleaved GPI-Anchored proteins from Schistosoma mansoni induces protection against challenge infection. Clin. Dev. Immunol. 2012: 962538.

McManus, DP, and Loukas, A, 2008: Current status of vaccines for schistosomiasis. Clin. Microbiol. Rev. 21, 1:225-42.

Nabih, I, Soliman, AM, 1986: Studies on fresh water snails, specific intermediate host for schistosomiasis. II. Isolation of total protein from native and irradiated snails. Cell Mol. Biol. 32: 315-7.

Nascimento, E, Leão, IC, Pereira, VR, Gomes, YM, Chikhlikar, P, et al, 2002: Protective immunity of single and multi-antigen DNA vaccines against schistosomiasis. Mem. Inst. Oswaldo Cruz. 97, 1105-9.

Pearce, EJ, MacDonald, AS, 2002: The immunobiology of schistosomiasis. Nat. Rev. Immunol. 2:499-511.

Pellegrino, J, Oliveira, CA, Faria, J, Cunha, AS, 1962: New approach to the screening of drugs in experimental schistosomiasis mansoni in mice. Am. J. Trop. Med. Hyg. 11:201-15.

Peters, PA, Warren, KS, 1969: A rapid method of infecting mice and other laboratory animals with Schistosoma mansoni: subcutaneous injection. Parasitol. 55, 3:55-8.

Pinto-Almeida, A, Mendes, T, de Oliveira, $\mathbf{R}$ N, Corrêa, A, Allegretti, S, et al, 2016: Morphological characteristics of Schistosoma mansoni PZQ-resistant and-susceptible strains are different in presence of praziquantel. Front Microbiol. Apr 26;7:594. doi: 10.3389/fmicb.2016.0059.

Ryll, R, Kumazawa, V, Vano, I, 2001: Immunological properties of Trehalose Dimycolate (cord factor) and other mycolic acid-containing glycolipids: A review. Microbiol. Immunol. 45, 12: 801-11.
Sharma, A, Haq, A, Ahmad, S, Lederer, E, 1985: Vaccination of rabbits against Entamoeba histolytica with aqueous suspensions of Trehalose-Dimycolate as the adjuvant. Infect. Immun. 85:634-7.

Sica, A, Mantovani, A, 2012: Macrophage plasticity and polarization: in vivo veritas. J. Clin. Invest. 122:787-95.

Steinauer, ML, 2009: The sex lives of parasites: Investigating the mating system and mechanisms of sexual selection of the human pathogen Schistosoma mansoni. Int. J. Parasitol. 39, 10:1157-63.

Stephenson, R, You, H, McManus, D, Toth, I, 2014: Schistosome vaccine adjuvants in preclinical and clinical research. Vaccines (Basel). 2, 3:654-85.

Teixeira de Melo, T, Araujo, JM, Campos de Sena, I, Carvalho Alves C, Araujo N, et al, 2013: Evaluation of the protective immune response induced in mice by immunization with $\mathrm{Sc}$ histosoma mansoni schistosomula tegument (Smteg) in association with CpG-ODN. Microbes Infect. 15, 1:28-36.

Tendler, M, Pinto, RM, Oliveira, LA, Gebara, G, Katz, N, 1986: Schistosoma mansoni: Vaccination with adult worm antigens. Int. J. Parasitol. 16, 4:347-52.

Vogel, FR, 2000: Improving vaccine performance with adjuvants. Clin. Infect. Dis. 30:26670.

WHO, 2020: Schistosomiasis: https://www.who .int/en/news-room/fact-sheets/detail/schistosomiasis.

WHO, 2018: WHO methods and data sources for country-level causes of death 2000-2016. Department of Information, Evidence and Research: Global Health Estimates Technical Paper WHO/ HIS/IER/GHE/2018.3.

WHO, 2013: Schistosomiasis: Progress Report 2001-2011, Strategic Plan 2012-2020.

Wilson, RA, Coulson, PS, Betts, C, Dowling, A, Smythies, LE, 1996: Impaired immunity and altered pulmonary responses in mice with a disrupted interferon- $\gamma$ receptor gene exposed to the irradiated Schistosoma mansoni vaccine. Immunol. 87:275-82.

Zhu, J, Xu, Z, Chen, X, Zhou, S, Zhang, W, et al, 2014: Parasitic antigens alter macrophage polarization during Schistosoma japonicum in fection in mice. Parasit. Vect. 7:122-33. 


\section{Explanation of figures}

Fig.1: SEM photos of SEA+TDM immunized mice: a- Sucker of $S$. mansoni male showed distortion, thinning and blebbing of sucker lips with disturbed direction of spines (x700), b- Tegument of $S$. mansoni male worm showed loss of normal tegument architecture, rough tegument, tubercular erosion and loss of spines with blebs formation (x1500), c- Gynaecophoric canal of S. mansoni male showed marked roughness with pores formation and widening of gynecophoric canal (x500), and d-S. mansoni female tegument showed sloughing, marked maceration and destruction of the tegument (x500).

Fig. 2: Comparison of groups regarding: a- Hepatic ova count, b- Intestinal ova count, and c-Oogram results.

Fig. 3: Comparison between groups: a- Mean granuloma number, b- Mean granuloma diameter, and c-Percentage of hepatic fibrosis.

Fig. 4: Trichrome stained hepatic tissue (Scale bar $=100 \mu \mathrm{m}$ ): a- Positive control group (GII) showing a large-sized granuloma surrounding ova (green arrow) with massive fibrosis (yellow arrow), and b- SEA+TDM (GV) showing a small-sized granuloma surrounding ova (green arrow) with massive fibrosis (yellow arrow)

Fig. 5: Comparison between mean H-scores of groups regarding: a- Hepatic expression of arginase-1, and b-Hepatic expression of iNOS.

Fig.6: Immune-histochemical reaction of hepatic tissue (Scale bar $=100 \mu \mathrm{m}$ ): a- Decreased expression of arginase-1 (red arrows) in positive GII limited to the periphery of a large-sized granuloma, b-Increased expression of arginase-1 (red arrows) in GV invading most of a smallsized granuloma, c- Increased expression of iNOS (red arrows) in positive GII invading most of a large-sized granuloma, and d-Decreased expression of iNOS (red arrows) in GV group limited to periphery of a small-sized granuloma.

Fig. 7: Comparison between groups regarding serum levels of: a- IgG after $1^{\text {st }}$ and booster dose of immunogens, b- IgG1 after 1 st and booster dose dose of immunogens, c- IgG2a after 1st and booster dose of immunogens, d- IL-10 after 1st \& booster dose of immunogens, e- IFN-y after 1 st and booster dose of immunogen.
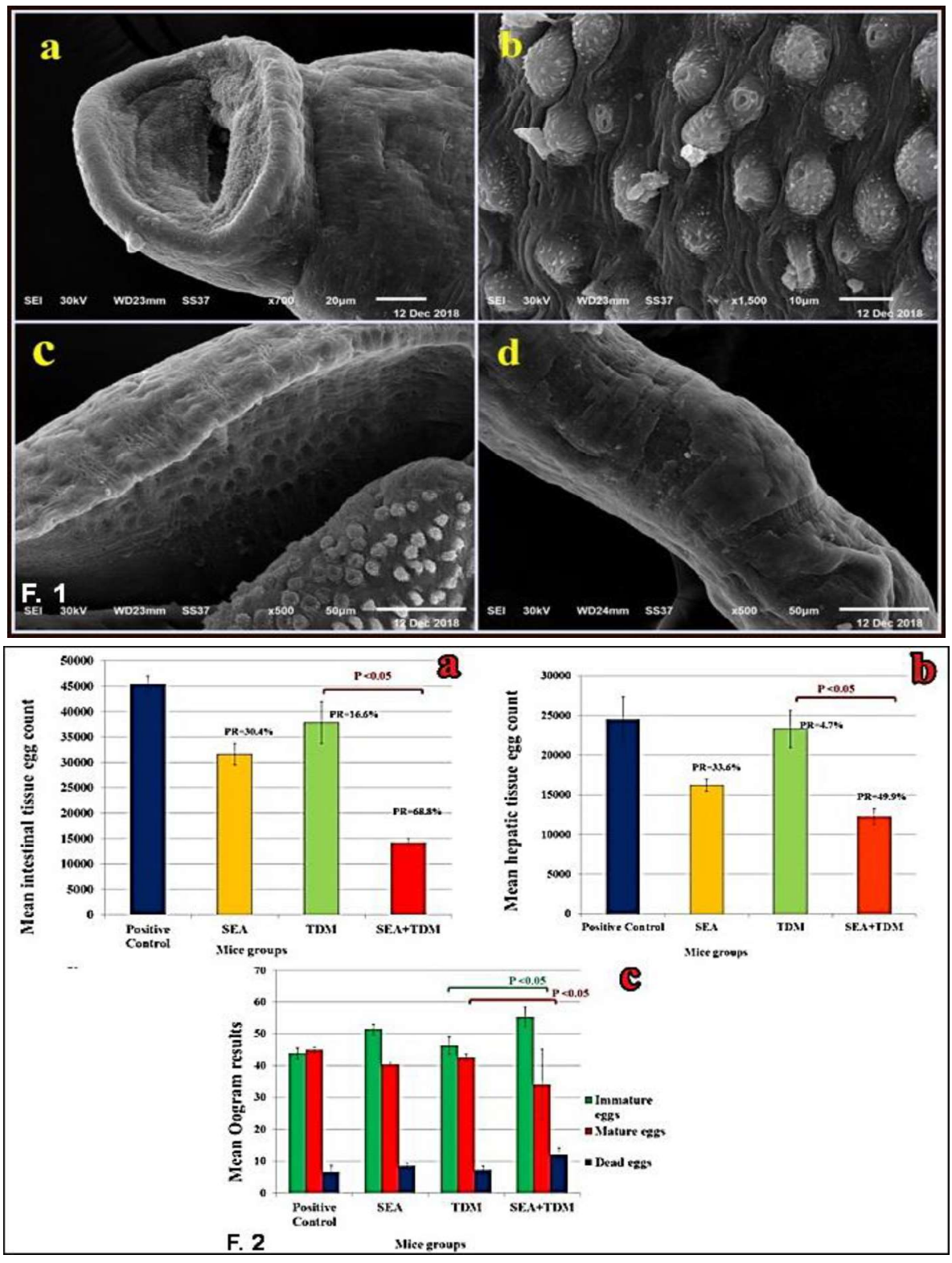

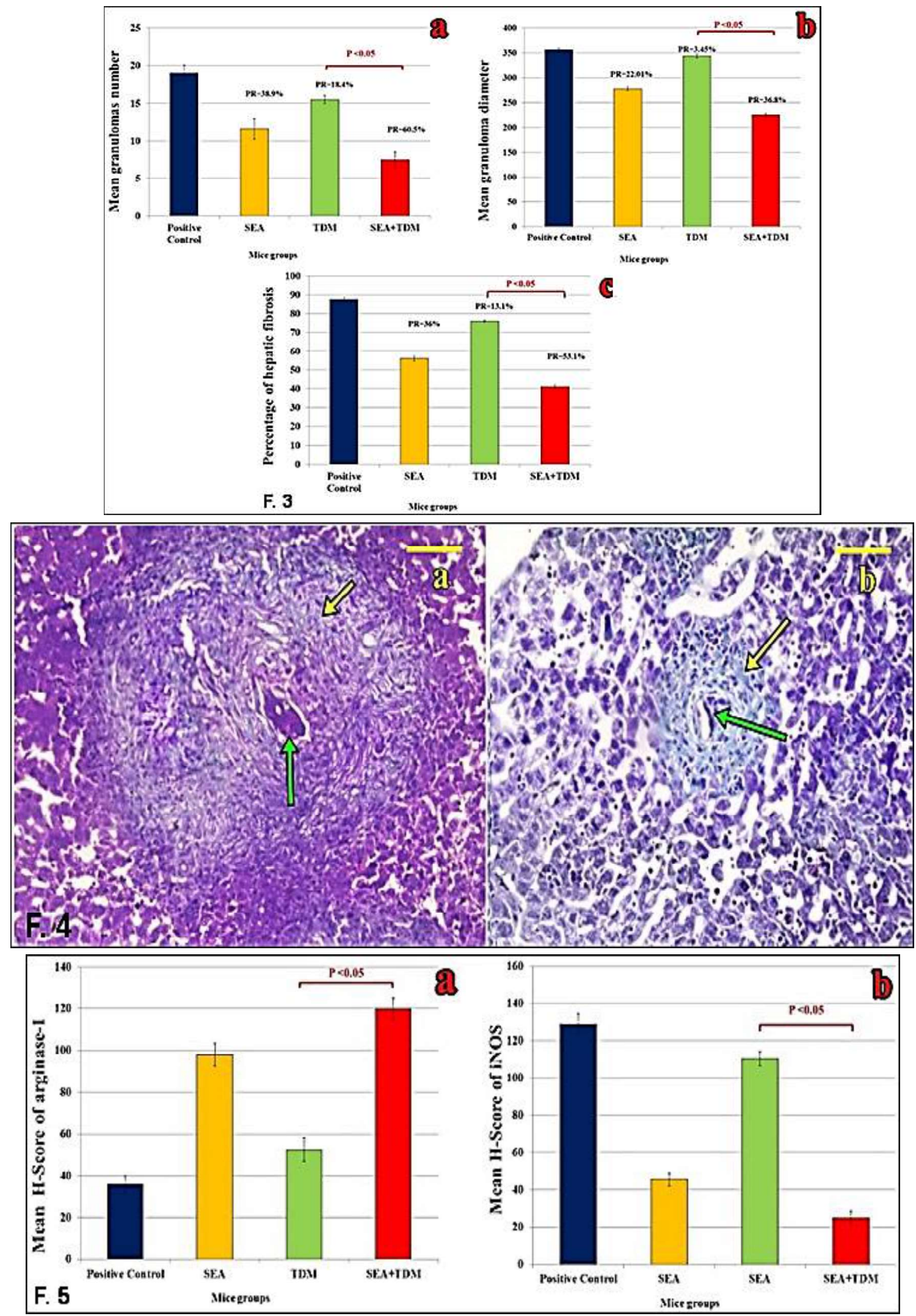

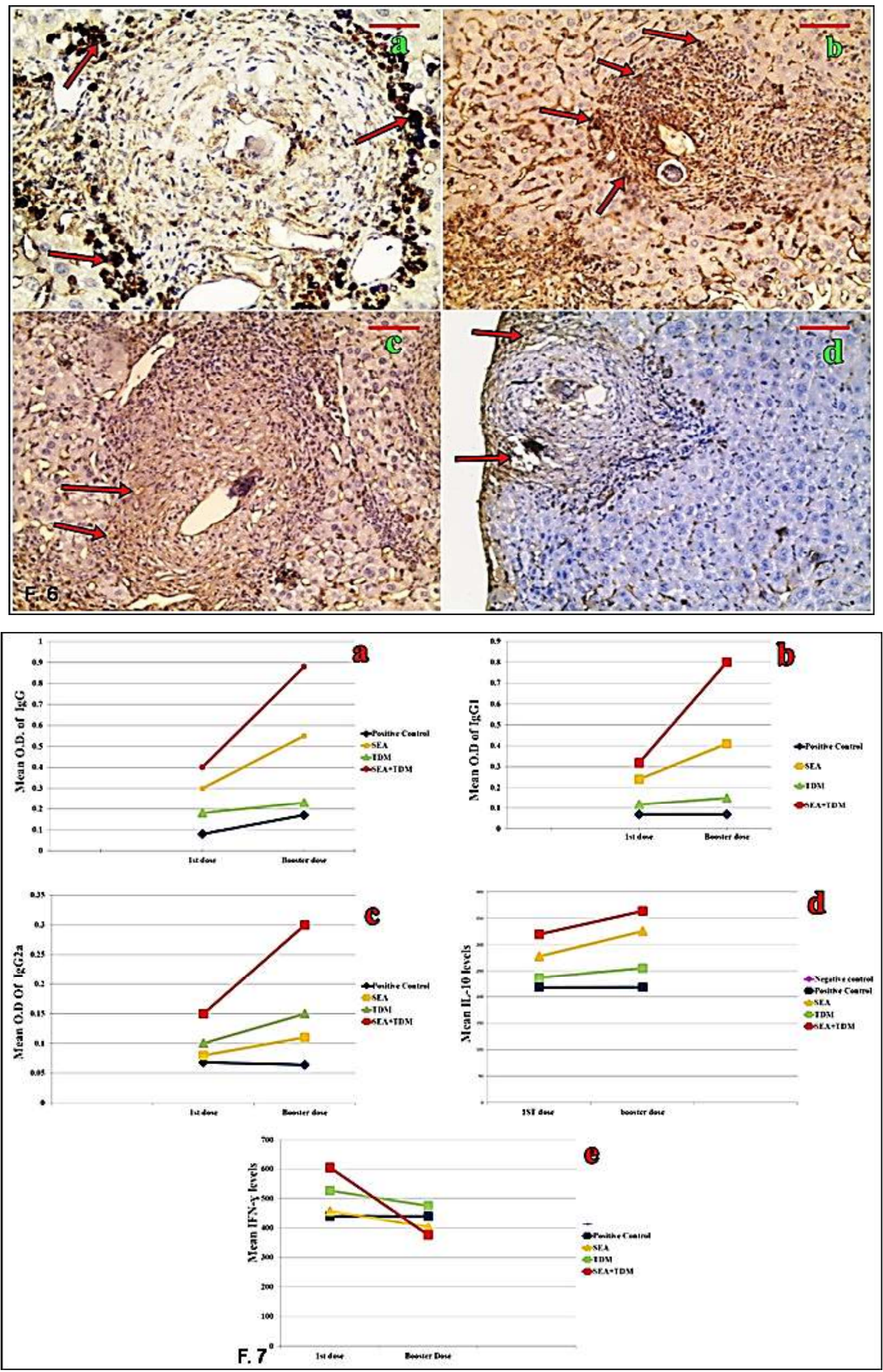